\title{
La producción científica en Chile y Latinoamérica
}

\author{
FRANCISCO CANO SCH. ${ }^{1}$, PAUL HARRIS D. ${ }^{1}$, LUISA SCHONHAUT B. ${ }^{1}$, FRANCISCA UGARTE P. ${ }^{1}$ \\ 1. Revista Chilena de Pediatría.
}

¿Es beneficioso, para un país cualquiera, destinar recursos al apoyo de su investigación científica?

¿Debiera Chile aumentar su inversión en ciencia y tecnología?

Con estas 2 simples preguntas se inicia el documento "Ciencia y Tecnología en Chile. Para que?” (http://www.conicyt.cl/documentos/CyTConicytparaque.pdf) publicado el año 2010 por la Comisión Nacional de Investigación Científica y Tecnológica, CONICYT.

Como antecedente se cita la declaración de la Agencia Estatal Norteamericana hace más de 50 años, que indicaba: "la investigación básica le entrega a la sociedad conocimientos invaluables y, considerando un período de tiempo, también retornos considerables a cambio de inversiones relativamente pequeñas. De hecho, los retornos son tan cuantiosos que resulta prácticamente innecesario justificar o evaluar la inversión realizada.”

Hoy, 50 años más tarde, nadie podría discutir que Investigación y Desarrollo (I+D) $(R \& D)$ son conceptos intimamente ligados, y que reflejan variados aspectos del desarrollo de un país, desde lo social, pasando por lo económico, hasta lo científico en sus áreas básica y clínica. La inversión actualmente es supervisada en forma detallada para que los recursos asignados se ajusten a una distribución justificada y se obtenga un rendimiento medible y en lo posible, recuperable. En Chile, el presupuesto de CONICYT, la principal agencia del Gobierno responsable de promover la ciencia y la tecnología se ha multiplicado por 3,3 en tér- minos reales en el período 2005-2010, lo que representa un crecimiento sin precedentes. En el documento CONICYT citado previamente, se pueden encontrar ejemplos de investigaciones financiadas por este fondo concursable, que dan cuenta de la amplia gama de proyectos y del impacto que representan para el país.

Uno de los resultados mas visibles de la Investigación en general, y médica en particular, son las publicaciones científicas originales. Para medir la importancia de la publicación científica se utilizan diferentes índices de citación, que miden el impacto de una investigación sobre futuras investigaciones. El principal de ellos, el Factor de Impacto (FI), esta contenido en el ISI (Institute for Scientific Information, Thomson Reuters) y se calcula en base al número de veces que una determinada publicación científica es citada por otras publicaciones durante los dos años siguientes a la aparición de la publicación evaluada. Cada año los resultados son publicados en el Journal of Citation Reports (JCR). Actualmente, otro índice de citación importante es elaborado a partir de Scopus, otra base de datos de publicaciones académicas.

De acuerdo a la Red de Indicadores de Ciencia y Tecnología-Iberoamericana e Interamericana-(RICYT), la producción científica en términos de publicaciones ISI en Chile es la más alta de la Región. Los datos del 2008 mostraron que el número de publicaciones ISI de Argentina fue 7 618, 31903 para Brasil, y 4251 para Chile. Cuando estos valores fueron ajustados por la población de los respectivos

Correspondencia a:

Francisco Cano Sch.

fcano@med.uchile.cl 
países Chile, Argentina y Brasil produjeron 25,3, 19,16, y 16,18 artículos ISI/105 habitantes respectivamente. Esta es una realidad creciente, lo cual se refleja en el hecho de que el porcentaje del producto bruto (GDP) invertido en Investigación y Desarrollo en latino América ha crecido en los últimos años desde un $0,45 \%$ en el año 1998 a un 0,68\% el año 2007. Nuestro país es el segundo de la Región después de Brasil, lo cual refleja el fortalecimiento que cada país está otorgando a esta actividad, que marca la frontera con el mundo desarrollado.

El informe RICYT 2011 sobre "Temas de Indicadores de Ciencia y Tecnología” (www. ricyt.org) incluye un capítulo sobre Indicadores de Producción Científica. En este capítulo se presenta un interesante estudio que evaluó el impacto que la colaboración con Europa tiene para los diferentes países de Latinoamérica a través de las publicaciones científicas de la Web of Science. Los principales resultados mostraron que durante el quinquenio 20022006 la producción científica de Latinoamérica fue de 191000 documentos, correspondiendo el 93\% de esta cifra a 4 países, Brasil, México, Argentina y Chile, y siendo Medicina Clínica el área mas importante en esta producción regional, con una cuarta parte del total. El perfil de colaboración mostró una asociación inversa entre el tamaño del país en términos de producción científica y Europa, principalmente España, Francia, Alemania, Reino Unido e Italia, sugiriendo una mayor autonomía de los grandes productores. Al analizar el impacto de la colaboración en términos de visibilidad (citaciones) se observó que ésta mejora la visibilidad de los países latinoamericanos para cada una de las áreas estudiadas. En las áreas con gran producción como Medicina Clínica, esta colaboración multiplicó por un factor de 2 ó 3 el número de citas por documento.

El total de documentos publicados por Brasil, México, Argentina y Chile en el período fue de 94 023, 37 807, 29799 y 15983 respectivamente. El porcentaje de incremento en el quinquenio fue de $42 \%$ para Chile, $38 \%$ para Brasil, 34\% para México y 12\% para Argentina, este último mostrando un porcentaje de incremento menor a la media. Al ajustar el nú- mero total de publicaciones por la población de cada pais, tenemos que, considerando para Brasil, México, Argentina y Chile una población aproximada de 194, 112, 41 y 17 mill/hbs respectivamente, la importancia de la producción científica se invierte, quedando Chile en el primer lugar de los 4 países de la región.

Al analizar la colaboración con Europa, España mostró ser el socio mas importante para la Región, seguido por Francia, Reino Unido, Alemania e Italia. España fue el principal socio colaborador con Argentina, Venezuela, Colombia, Uruguay y Chile, en tanto que Francia destaca por la coproducción asociada con Brasil. Alemania mostró su mayor impacto en la coproducción científica con 2 países, Chile y Costa Rica, en tanto que Reino Unido fue el principal colaborador con Colombia. Estos datos resultan de la mayor importancia al analizar la formulación de Proyectos Fondecyt u otros, en el marco de la colaboración internacional.

Cómo se inserta nuestra publicación científica "Revista Chilena de Pediatría” en el contexto Regional analizado?

Actualmente, con 421 números de la revista en línea, www.scielo.cl, y una cifra de 9245000 visitas a los artículos en los últimos 10 años, 2002-2012, sabemos de la importancia que representa para la pediatría nacional, solamente superada por la Revista Médica de Chile que agrupando a una amplia gamma de especialistas muestra 13 millones de visitas en el mismo período. El impacto que las cifras muestran supera nuestras fronteras y expectativas. Es así como en el período julio 2010-junio 2011, un 20\% de los artículos originales publicados provenían de otros países de Latinoamérica e Iberoamérica, cifra que se mantiene hasta la fecha. Contamos para ello con la desinteresada e invaluable colaboración de aproximadamente 70 evaluadores, los cuales en el formato digital OJS implementado a partir de un proyecto CONICYT hace 2 años ha permitido una manejo altamente eficiente del trabajo editorial en la Revista. Con el apoyo del recientemente formado cuerpo de asesores editoriales internacionales, hemos iniciado un camino de optimización de la publicación que esperamos en mediano plazo nos permita vi- 
sualizarnos en el SCI (Science Citation Index, Thomson Reuters). En este continuo esfuerzo de modernización para estar disponibles en todo momento para nuestros lectores en Chile y en el extranjero, desde el número 1 del año 2012, Revista Chilena de Pediatría podrá ser leida en formato electrónico de portátiles como tablets, entregando una portabilidad que en un corto futuro sin duda constituirá una importante fuente de consulta para todos los medios actualmente disponibles en papel.

\section{¿Cuál sería entonces el desafío prioritario?}

En sus orígenes, hace 82 años, la Revista Chilena de Pediatría publicaba en su mayoría artículos de reflexión y análisis de casos clínicos, los que de a poco se convirtieron en series clínicas y fueron incorporando cada vez más el pensamiento científico y la evidencia. Un análisis sobre las publicaciones de nuestra revista en el quinquenio 2001-2006 mostró que el 35\% de los artículos publicados eran originales (Harris P. y cols, . Rev Chil Pediatr 2007; 78). No obstante, al analizar el tipo artículos originales publicados, podemos notar que falta producir investigación de mayor complejidad, como son los estudios clínicos controlados, aleatorizados, doble ciego o los estudios controlados abiertos, caso-control o seguimientos prospectivos de cohorte, ya que son los que generan la más contundente evidencia científica. Según un análisis del decenio 1994-2003 (Castillo C. y cols, Rev Chil Pediatr 2005; 76, 2009; 80) este tipo de estudios son de baja fre- cuencia en la producción nacional, con una tendencia estacionaria en la última década. No obstante, al compararnos con la realidad latinoamericana, podemos percatarnos de nuestro gran potencial, ya que Chile se encuentra entre los 4 países con mayor número de publicaciones pediátricas de estudios clínicos controlados aleatorios, siendo superado por Brasil y México, tendencia que se invierte al ajustar las cifras por número de habitantes, pasando a ser Chile y Argentina los más productivos, pero aún lejos de los países desarrollados.

Si bien hay mucho espacio para mejorar la visibilidad de la investigación pediátrica en Chile a través de nuestra Revista, aún queda mucho camino por avanzar, tarea que debemos asumir como miembros de esta Sociedad Chilena de Pediatría, y como profesionales involucrados en el manejo y cuidado del niño tanto en el diagnóstico y tratamiento de su enfermedad como en su prevención.

En nuestro país de acuerdo a lo analizado, encontramos amplias oportunidades para desarrollar I+D en medicina, a un nivel Regional que de acuerdo a las cifras está en una situación que podemos considerar altamente favorable. Ahora es responsabilidad de todos el aquilatar las preguntas de investigación relevantes y dar respuestas a ellas en forma científica. Finalmente, si no lo hacemos nosotros, estaremos faltando a una de las más grandes responsabilidades que la sociedad nos ha encomendado, cual es responder a los desafíos venideros y anticiparnos a lo que será la Pediatría de los próximos decenios. La invitación está abierta.

\section{A LOS AUTORES}

Comunicamos a los autores de los artículos presentados para publicación en Revista Chilena de Pediatría, que ha concluido exitosamente el Proyecto Editorial del Fondo de Publicación de Revistas Científicas CONICYT 2008-2009 "Revista Chilena de Pediatría: De las Publicaciones en Papel a un Proceso Editorial On-Line basado en Open Journal System". Por lo tanto, solicitamos a los autores de los artículos presentados a partir de ahora, que ingresen a www.revistachilenadepediatria.cl, se registren e identifiquen de acuerdo a las instrucciones, y procedan a ingresar su contribución al sistema editorial electrónico según se detalla.

La Sociedad Chilena de Pediatria y Revista Chilena de Pediatría se enorgullecen de poner a disposición de nuestros colaboradores esta moderna herramienta editorial, en forma pionera entre las publicaciones biomédicas de nuestro país.

El Editor 